\title{
ASYMPTOTIC FORMULAS FOR TWO CONTINUED FRACTIONS IN RAMANUJAN'S LOST NOTEBOOK
}

\author{
Bruce C. Berndt and Jaebum Sohn
}

\begin{abstract}
On page 45 of his lost notebook, Ramanujan recorded two asymptotic formulas for two continued fractions involving the Riemann zeta function and Dirichlet $L$-functions. In this paper, we prove a more general theorem and derive Ramanujan's claims as a corollary of our theorem
\end{abstract}

\section{Introduction}

Several elegant $q$-continued fractions have representations as $q$-products. The most famous one, of course, is the Rogers-Ramanujan continued fraction defined for $|q|<1$, by

$$
R(q):=\frac{q^{1 / 5}}{1}+\frac{q}{1}+\frac{q^{2}}{1}+\frac{q^{3}}{1}+\cdots=q^{1 / 5} \frac{\left(q ; q^{5}\right)_{\infty}\left(q^{4} ; q^{5}\right)_{\infty}}{\left(q^{2} ; q^{5}\right)_{\infty}\left(q^{3} ; q^{5}\right)_{\infty}} .
$$

Here we use the notation for continued fractions,

$$
b_{0}+\frac{a_{1}}{b_{1}+\frac{a_{2}}{b_{2}+\frac{a_{3}}{b_{3}+\cdots}}}=b_{0}+\frac{a_{1}}{b_{1}}+\frac{a_{2}}{b_{2}}+\frac{a_{3}}{b_{3}}+\cdots
$$

Also we employ the customary notation

$$
(a ; q)_{\infty}:=\prod_{k=0}^{\infty}\left(1-a q^{k}\right), \quad|q|<1 .
$$

In the sequel, we also use the notation

$$
\left(a_{1}, a_{2}, \ldots, a_{n} ; q\right)_{\infty}:=\left(a_{1} ; q\right)_{\infty}\left(a_{2} ; q\right)_{\infty} \cdots\left(a_{n} ; q\right)_{\infty} .
$$

First author's research was sponsored by grant MDA904-00-1-0015 from theNational Security Agency.

2000 Mathematics Subject Classification: 11B65, 11A55, 11M06 
Other $q$-products having continued fraction representations include, for $|q|<1$,

$$
\begin{aligned}
\frac{\left(q^{2} ; q^{3}\right)_{\infty}}{\left(q ; q^{3}\right)_{\infty}} & =\frac{1}{1}-\frac{q}{1+q}-\frac{q^{3}}{1+q^{2}}-\frac{q^{5}}{1+q^{3}}-\frac{q^{7}}{1+q^{4}}-\ldots, \\
\frac{\left(q^{3} ; q^{4}\right)_{\infty}}{\left(q ; q^{4}\right)_{\infty}} & =\frac{1}{1}-\frac{q}{1+q^{2}}-\frac{q^{3}}{1+q^{4}}-\frac{q^{5}}{1+q^{6}}-\frac{q^{7}}{1+q^{8}}-\ldots, \\
q^{1 / 2} \frac{\left(q ; q^{8}\right)_{\infty}\left(q^{7} ; q^{8}\right)_{\infty}}{\left(q^{3} ; q^{8}\right)_{\infty}\left(q^{5} ; q^{8}\right)_{\infty}} & =\frac{q^{1 / 2}}{1+q}+\frac{q^{2}}{1+q^{3}}+\frac{q^{4}}{1+q^{5}}+\frac{q^{6}}{1+q^{7}}+\ldots=: H(q) .
\end{aligned}
$$

On page 45 of his lost notebook [21], Ramanujan recorded two asymptotic formulas for two continued fractions involving the Riemann zeta function and Dirichlet $L$-functions. These continued fractions are equivalent to (1.2) and (1.3) after a change of variable. They are among the most interesting continued fractions discovered by Ramanujan. The continued fraction (1.3) also converges for $|q|>1$, and it converges to

$$
\frac{\left(q^{-3} ; q^{-4}\right)_{\infty}}{\left(q^{-1} ; q^{-4}\right)_{\infty}}
$$

which provides a beautiful example of symmetry. The continued fraction (1.2) is the most difficult to prove of all of Ramanujan's continued fractions and does not seem to fit in the same hierarchy as the other $q$-continued fractions found by Ramanujan. Other unusual properties of this continued fraction can also be found on page 45 of [21]. For a further discussion of these continued fractions, see [9, pp. 46-49]. We state one of the two results on page 45.

Let $\zeta(s)=\sum_{n=1}^{\infty} n^{-s}$, Re $s>1$, denote the Riemann zeta-function, and let $L(s, \chi)=\sum_{n=1}^{\infty} \chi(n) n^{-s}$ denote the Dirichlet $L$-function associated with the character $\chi(n)=\left(\frac{n}{3}\right)$, the Legendre symbol. For each integer $n \geqslant 2$, let

$$
a_{n}=\frac{4 \Gamma(n) \zeta(n) L(n+1, \chi)}{(2 \pi / \sqrt{3})^{2 n+1}} .
$$

Then, for $x>0$,

$$
\frac{(3 x)^{1 / 3}}{1}-\frac{1}{1+e^{x}}-\frac{1}{1+e^{2 x}}-\frac{1}{1+e^{3 x}}-\cdots=\frac{\Gamma\left(\frac{1}{3}\right)}{\Gamma\left(\frac{2}{3}\right)} e^{G(x)},
$$

where, as $x \rightarrow 0+$,

$$
G(x) \sim a_{2} x^{2}+a_{4} x^{4}+a_{6} x^{6}+\cdots
$$

In particular,

$$
a_{2}=\frac{1}{108}, \quad a_{4}=\frac{1}{4320}, \quad a_{6}=\frac{1}{38880} .
$$

In Section 2, we prove a more general theorem for odd characters $\chi$, and in Section 3 we derive Ramanujan's claims as corollaries of our theorem. In Section 4, we 
prove a general theorem for even characters $\chi$, and give asymptotic formulas for the Rogers-Ramanujan continued fraction and the Göllnitz-Gordon continued fraction stated in (1.4) as $q \rightarrow 1-$. L. Richmond and G. Szekeres [22] gave asymptotic formulas for the Rogers-Ramanujan and the Göllnitz-Gordon continued fractions as $q \rightarrow 0+$.

\section{The Main Theorem}

We need a form of Stirling's formula; see [4, p. 539] or [11, p. 224].

Lemma 2.1. As $|t| \rightarrow \infty$,

$$
|\Gamma(\sigma+i t)| \sim \sqrt{2 \pi} e^{-\pi|t| / 2}|t|^{\sigma-\frac{1}{2}}
$$

uniformly in any fixed vertical strip $\alpha \leqslant \sigma \leqslant \beta$.

Theorem 2.2. Let $k$ be a positive integer greater than or equal to 3, and let $L(s, \chi)$ denote the Dirichlet $L$-function associated with $\chi(n)$, a primitive real non-principal odd character mod $k$. Then as $x \rightarrow 0+$,

$$
(x k)^{-\frac{M_{1}(\chi)}{k}} \prod_{n=1}^{k-1}\left(e^{-n x} ; e^{-k x}\right)_{\infty}^{-\chi(n)}=\prod_{n=1}^{k-1} \Gamma\left(\frac{n}{k}\right)^{\chi(n)} e^{G(x)},
$$

where

$$
M_{1}(\chi)=\sum_{n=1}^{k-1} \chi(n) n
$$

and

$$
G(x) \sim a_{2} x^{2}+a_{4} x^{4}+a_{6} x^{6}+\cdots,
$$

with

$$
a_{\nu}=\frac{4 \Gamma(\nu)}{(2 \pi / \sqrt{k})^{2 \nu+1}} \zeta(\nu) L(\nu+1, \chi)
$$

Also, as $x \rightarrow 0+$,

$$
\text { the minimum value of } a_{\nu} x^{\nu} \sim \frac{k}{\pi} \sqrt{\frac{2 x}{\pi}} e^{-\frac{4 \pi^{2}}{k x}} .
$$

Proof. Let

$$
P(x):=\prod_{n=1}^{k-1}\left(e^{-n x} ; e^{-k x}\right)_{\infty}^{-\chi(n)}
$$

Then, for $x>0$,

$$
\begin{aligned}
f(x):=\log P(x) & =-\sum_{n=1}^{\infty} \chi(n) \log \left(1-e^{-n x}\right) \\
& =\sum_{n=1}^{\infty} \chi(n) \sum_{m=1}^{\infty} \frac{e^{-n m x}}{m} .
\end{aligned}
$$


Thus, inverting the order of summation and integration by absolute convergence, we find that, for $x>0$,

$$
\begin{aligned}
\int_{0}^{\infty} f(x) x^{s-1} d x & =\int_{0}^{\infty} \sum_{n=1}^{\infty} \chi(n) \sum_{m=1}^{\infty} \frac{e^{-n m x}}{m} x^{s-1} d x \\
& =\sum_{n=1}^{\infty} \sum_{m=1}^{\infty} \frac{\chi(n)}{m} \int_{0}^{\infty} e^{-n m x} x^{s-1} d x \\
& =\sum_{n=1}^{\infty} \sum_{m=1}^{\infty} \frac{\chi(n)}{m} \int_{0}^{\infty} e^{-u}\left(\frac{u}{n m}\right)^{s-1} \frac{d u}{n m} \\
& =\sum_{n=1}^{\infty} \sum_{m=1}^{\infty} \chi(n) \frac{1}{m^{s+1}} \frac{1}{n^{s}} \int_{0}^{\infty} e^{-u} u^{s-1} d u \\
& =\sum_{n=1}^{\infty} \sum_{m=1}^{\infty} \chi(n) \frac{1}{m^{s+1}} \frac{1}{n^{s}} \Gamma(s) \\
& =\Gamma(s) \zeta(s+1) L(s, \chi) .
\end{aligned}
$$

By Mellin's inversion formula [24, p. 7],

$$
f(x)=\frac{1}{2 \pi i} \int_{c-i \infty}^{c+i \infty} \Gamma(s) \zeta(s+1) L(s, \chi) x^{-s} d s, \quad c>1
$$

Consider now

$$
I_{C_{M, T}}:=\frac{1}{2 \pi i} \int_{C_{M, T}} \Gamma(s) \zeta(s+1) L(s, \chi) x^{-s} d s
$$

where $M=2 N+\frac{1}{2}, N$ is any positive integer, and $C_{M, T}$ is the positively oriented rectangle with corners at $(c, i T),(-M, i T),(-M,-i T)$, and $(c,-i T)$, where $T$ is any positive number.

Recall that $\Gamma(s)$ has a simple pole at $s=-n$ with residue $(-1)^{n} / n$ !, for each nonnegative integer $n$. Recall also that $\zeta(s)$ has a simple pole at $s=1$ with residue 1 , and that $\zeta(-2 n)=0$ for each positive integer $n$ [25, pp. 16, 19]. Furthermore, since $\chi$ is odd, $L(-2 n-1, \chi)=0$ for each nonnegative integer $n[12$, p. 71]. Hence, the integrand of (2.6) has simple poles at $s=-2,-4,-6, \ldots,-2 N$ and a double pole at $s=0$ on the interior of $C_{M, T}$.

Using the expansions [16, p. 944], [25, p. 16],

$$
\begin{aligned}
\Gamma(s) & =\frac{1}{s}-\gamma+\cdots, \\
\zeta(s+1) & =\frac{1}{s}+\gamma+\cdots, \\
x^{-s} & =e^{-s \log x}=1-s \log x+\cdots,
\end{aligned}
$$


and

$$
L(s, \chi)=L(0, \chi)+L^{\prime}(0, \chi) s+\cdots,
$$

where $\gamma$ denotes Euler's constant, we find that

$$
\begin{aligned}
& \Gamma(s) \zeta(s+1) L(s, \chi) x^{-s} \\
= & \left(\frac{1}{s}-\gamma+\cdots\right)\left(\frac{1}{s}+\gamma+\cdots\right)(1-s \log x+\cdots)\left(L(0, \chi)+L^{\prime}(0, \chi) s+\cdots\right) .
\end{aligned}
$$

Hence, the residue at $s=0$ is

$$
\begin{aligned}
R_{0} & :=-L(0, \chi) \log x+L^{\prime}(0, \chi)+\gamma L(0, \chi)-\gamma L(0, \chi) \\
& =-L(0, \chi) \log x+L^{\prime}(0, \chi) .
\end{aligned}
$$

The residue at $s=-2 n, n \geqslant 1$, is

$$
R_{-2 n}:=\frac{1}{(2 n) !} \zeta(1-2 n) L(-2 n, \chi) x^{2 n}
$$

Next, we estimate the integrals along the horizontal sides. First, from [25, p. 81], for $-M \leqslant \sigma \leqslant c$,

$$
\zeta(1+\sigma \pm i T)=O\left(T^{M+\frac{1}{2}}\right)
$$

as $T \rightarrow \infty$. Also from [6, pp. 270-273] and the Phragmén-Lindelöf theorem, for $-M \leqslant \sigma \leqslant c$,

$$
L(\sigma \pm i T, \chi)=O\left(T^{M+1}\right)
$$

as $T \rightarrow \infty$.

Hence from Lemma 2.1, (2.9), and (2.10), we have

$$
\begin{aligned}
& \int_{-M}^{c} \Gamma(\sigma \pm i T) \zeta(1+\sigma \pm i T) L(\sigma \pm i T, \chi) x^{-\sigma \mp i T} d \sigma \\
= & O\left(\int_{-M}^{c} e^{-\pi T / 2} T^{2 M+c+1} x^{M} d \sigma\right)=o(1),
\end{aligned}
$$

as $T \rightarrow \infty$.

Thus, having let $T \rightarrow \infty$, there remains to examine

$$
\int_{-\infty}^{\infty} \Gamma(-M+i t) \zeta(1-M+i t) L(-M+i t, \chi) x^{M-i t} d t
$$

Now by using the elementary identity $\sin ^{2}(x+i y)=\sin ^{2} x+\sinh ^{2} y$, the reflection formula

$$
\Gamma(s) \Gamma(1-s)=\frac{\pi}{\sin \pi s},
$$


and Lemma 2.1, we deduce that

$$
\begin{aligned}
\Gamma(-M+i t) & =\frac{\pi}{\sin \pi(-M+i t) \Gamma(1+M-i t)} \\
& =\frac{\pi}{\left\{\sin ^{2} \pi(-M)+\sinh ^{2} \pi t\right\}^{\frac{1}{2}} \Gamma(1+M-i t)} \\
& =O\left(\frac{1}{e^{\pi|t|} e^{-\pi|t| / 2}|t|^{M+\frac{1}{2}}}\right) \\
& =O\left(|t|^{-M-\frac{1}{2}} e^{-\pi|t| / 2}\right)
\end{aligned}
$$

as $|t| \rightarrow \infty$.

Thus by (2.9), (2.10), and the calculation above,

$$
\begin{aligned}
& \int_{1}^{ \pm \infty} \Gamma(-M+i t) \zeta(1-M+i t) L(-M+i t, \chi) x^{M-i t} d t \\
= & O\left(\int_{1}^{ \pm \infty} e^{-\pi|t| / 2}|t|^{M+1} x^{M} d t\right) \\
= & O\left(x^{M}\right),
\end{aligned}
$$

as $x \rightarrow 0+$. Hence, as $x \rightarrow 0+$, by (2.6), the residue theorem, (2.5), (2.7), (2.8), (2.11), and (2.13),

$$
\begin{aligned}
f(x)= & -L(0, \chi) \log x+L^{\prime}(0, \chi) \\
& +\sum_{n=1}^{N} \frac{1}{(2 n) !} \zeta(1-2 n) L(-2 n, \chi) x^{2 n}+O\left(x^{2 N+\frac{1}{2}}\right) .
\end{aligned}
$$

Since $\chi$ is an odd character, the functional equation for $L(s, \chi)$ is given by $[12, \mathrm{p}$. 71]

$$
L(s, \chi)=\left(\frac{\pi}{k}\right)^{s-\frac{1}{2}} \frac{\Gamma\left(1-\frac{1}{2} s\right)}{\Gamma\left(\frac{1}{2}(s+1)\right)} L(1-s, \chi) .
$$

Now from (2.12), we have

$$
\begin{aligned}
\Gamma\left(\frac{1}{2}-n\right) & =\frac{\pi}{\sin \pi\left(\frac{1}{2}-n\right) \Gamma\left(n+\frac{1}{2}\right)} \\
& =\frac{\pi}{(-1)^{n} \Gamma\left(n+\frac{1}{2}\right)} \\
& =\frac{\pi(-1)^{n} 2^{n}}{1 \cdot 3 \cdots(2 n-1) \sqrt{\pi}} \\
& =\frac{\sqrt{\pi}(-1)^{n} 2^{2 n} n !}{(2 n) !}
\end{aligned}
$$


since $\Gamma(1 / 2)=\sqrt{\pi}$. Thus, from (2.15) and (2.16),

$$
\begin{aligned}
L(-2 n, \chi) & =\left(\frac{\pi}{k}\right)^{-2 n-\frac{1}{2}} \frac{\Gamma(n+1)}{\Gamma\left(\frac{1}{2}-n\right)} L(2 n+1, \chi) \\
& =\left(\frac{\pi}{k}\right)^{-2 n-\frac{1}{2}} \frac{n !(2 n) !}{\sqrt{\pi}(-1)^{n} 2^{2 n} n !} L(2 n+1, \chi) \\
& =\left(\frac{k}{\pi}\right)^{2 n+\frac{1}{2}} \frac{(-1)^{n}(2 n) !}{2^{2 n} \sqrt{\pi}} L(2 n+1, \chi) .
\end{aligned}
$$

By the functional equation for $\zeta(s)$ [25, p. 16, Eq. (2.1.8)],

$$
\zeta(1-2 n)=\frac{2(-1)^{n}(2 n-1) !}{(2 \pi)^{2 n}} \zeta(2 n) .
$$

Thus, using (2.17) and (2.18) in (2.14), we find that

$$
\begin{aligned}
f(x)= & -L(0, \chi) \log x+L^{\prime}(0, \chi) \\
& +\sum_{n=1}^{N} \frac{1}{(2 n) !} \frac{2(-1)^{n}(2 n-1) !}{(2 \pi)^{2 n}} \zeta(2 n)\left(\frac{k}{\pi}\right)^{2 n+\frac{1}{2}} \frac{(-1)^{n}(2 n) !}{2^{2 n} \sqrt{\pi}} L(2 n+1, \chi) x^{2 n} \\
& +O\left(x^{2 N+\frac{1}{2}}\right) \\
= & -L(0, \chi) \log x+L^{\prime}(0, \chi) \\
& +\sum_{n=1}^{N} \frac{4 \Gamma(2 n)}{(2 \pi / \sqrt{k})^{4 n+1}} \zeta(2 n) L(2 n+1, \chi) x^{2 n}+O\left(x^{2 N+\frac{1}{2}}\right) .
\end{aligned}
$$

Next from the functional equation (2.15),

$$
L(0, \chi)=\frac{\sqrt{k}}{\pi} L(1, \chi) \text {. }
$$

But from [10, p. 336, Thm. 3],

$$
L(1, \chi)=-\frac{\pi \sqrt{k}}{k^{2}} M_{1}(\chi),
$$

where $M_{1}(\chi)$ is defined by (2.2). Thus

$$
L(0, \chi)=\frac{\sqrt{k}}{\pi}\left(-\frac{\pi \sqrt{k}}{k^{2}}\right) M_{1}(\chi)=-\frac{M_{1}(\chi)}{k} .
$$

By the functional equation (2.15) and the chain rule, after simplifying, we find that

$$
\begin{aligned}
L^{\prime}(s, \chi)= & \left(\frac{\pi}{k}\right)^{s-\frac{1}{2}} \frac{\Gamma\left(1-\frac{1}{2} s\right)}{\Gamma\left(\frac{1}{2}(1+s)\right)} L(1-s, \chi) \\
& \times\left(\log \frac{\pi}{k}-\frac{1}{2} \psi\left(1-\frac{1}{2} s\right)-\frac{1}{2} \psi\left(\frac{1}{2}(s+1)\right)-\frac{L^{\prime}(1-s, \chi)}{L(1-s, \chi)}\right),
\end{aligned}
$$


where $\psi(s)=\Gamma^{\prime}(s) / \Gamma(s)$. Hence at $s=0$,

$$
L^{\prime}(0, \chi)=\left(\frac{\pi}{k}\right)^{-\frac{1}{2}} \frac{\Gamma(1)}{\Gamma\left(\frac{1}{2}\right)} L(1, \chi)\left(\log \frac{\pi}{k}-\frac{1}{2} \psi(1)-\frac{1}{2} \psi\left(\frac{1}{2}\right)-\frac{L^{\prime}(1, \chi)}{L(1, \chi)}\right) .
$$

¿From [1, p. 258],

$$
\psi(1)=-\gamma \quad \text { and } \quad \psi\left(\frac{1}{2}\right)=-\gamma-2 \log 2
$$

where $\gamma$ is Euler's constant.Thus, from (2.22), (2.20), and (2.23),

$$
\begin{aligned}
L^{\prime}(0, \chi) & =\left(\frac{\pi}{k}\right)^{-\frac{1}{2}} \frac{1}{\sqrt{\pi}}\left(-\frac{\pi \sqrt{k}}{k^{2}}\right) M_{1}(\chi)\left(\log \frac{\pi}{k}+\frac{1}{2} \gamma+\frac{1}{2} \gamma+\log 2-\frac{L^{\prime}(1, \chi)}{L(1, \chi)}\right) \\
& =-\frac{M_{1}(\chi)}{k}\left(\log \frac{2 \pi}{k}+\gamma\right)-\frac{\sqrt{k}}{\pi} L^{\prime}(1, \chi) .
\end{aligned}
$$

By a theorem of C. Deninger [13, p. 182],

$$
L^{\prime}(1, \chi)=-\frac{\pi}{\sqrt{k}}\left((\gamma+\log 2 \pi) \frac{M_{1}(\chi)}{k}+\sum_{n=1}^{k-1} \chi(n) \log \left(\Gamma\left(\frac{n}{k}\right)\right)\right) .
$$

Thus, by (2.24) and (2.25),

$$
\begin{aligned}
& L^{\prime}(0, \chi)=-\frac{M_{1}(\chi)}{k} \log 2 \pi+\frac{M_{1}(\chi)}{k} \log k-\gamma \frac{M_{1}(\chi)}{k} \\
& \quad+\frac{\sqrt{k}}{\pi} \frac{\pi}{\sqrt{k}}\left(\gamma \frac{M_{1}(\chi)}{k}+\frac{M_{1}(\chi)}{k} \log 2 \pi+\sum_{n=1}^{k-1} \chi(n) \log \left(\Gamma\left(\frac{n}{k}\right)\right)\right) \\
&=\frac{M_{1}(\chi)}{k} \log k+\sum_{n=1}^{k-1} \chi(n) \log \left(\Gamma\left(\frac{n}{k}\right)\right) .
\end{aligned}
$$

Hence, from (2.19), (2.21), and (2.26),

$$
\begin{aligned}
f(x)=\frac{M_{1}(\chi)}{k} & \log x k+\sum_{n=1}^{k-1} \chi(n) \log \left(\Gamma\left(\frac{n}{k}\right)\right) \\
& +\sum_{n=1}^{N} \frac{4 \Gamma(2 n)}{(2 \pi / \sqrt{k})^{4 n+1}} \zeta(2 n) L(2 n+1, \chi) x^{2 n}+O\left(x^{2 N+\frac{1}{2}}\right),
\end{aligned}
$$

which, upon exponentiation, completes the proof of (2.1).

To prove (2.4), let

$$
g(t)=\frac{4 \Gamma(t) x^{t}}{(2 \pi / \sqrt{k})^{2 t+1}}=\frac{4 \Gamma(t) x^{t}}{c^{2 t+1}}
$$


where $c=2 \pi / \sqrt{k}$. We want to minimize $g(t)$. By the chain rule

$$
\begin{aligned}
g^{\prime}(t) & =\frac{4 \Gamma^{\prime}(t) x^{t}}{c^{2 t+1}}+\frac{4 \Gamma(t) x^{t} \log x}{c^{2 t+1}}-\frac{8 \Gamma(t) x^{t} \log c}{c^{2 t+1}} \\
& =\frac{4 \Gamma(t) x^{t}}{c^{2 t+1}}\left(\frac{\Gamma^{\prime}(t)}{\Gamma(t)}+\log x-2 \log c\right)=0 .
\end{aligned}
$$

So $g(t)$ has a minimum value at the point $t$ that satisfies

$$
\psi(t)=\log \left(\frac{c^{2}}{x}\right)
$$

where $\psi(t)=\Gamma^{\prime}(t) / \Gamma(t)$. But from [1, p. 259], as $t \rightarrow \infty$,

$$
\psi(t) \sim \log t,
$$

and therefore

$$
t=\frac{c^{2}}{x}
$$

Thus by Stirling's formula [1, p. 257] and the calculation above, the minimum value of $a_{t} x^{t}$ is, as $x \rightarrow 0+$,

$$
\begin{aligned}
\frac{4 \sqrt{2 \pi} t^{t-1 / 2} e^{-t} x^{t}}{c^{2 t+1}} & \sim \frac{4 \sqrt{2 \pi}\left(\frac{c^{2}}{x}\right)^{c^{2} / x-1 / 2} e^{-c^{2} / x} x^{c^{2} / x}}{c^{2 c^{2} / x+1}} \\
& =\frac{4 \sqrt{2 \pi} c^{2 c^{2} / x-1} x^{1 / 2} e^{-c^{2} / x}}{c^{2 c^{2} / x+1}} \\
& =\frac{4 \sqrt{2 \pi} \sqrt{x} e^{-c^{2} / x}}{c^{2}} \\
& =\frac{k}{\pi} \sqrt{\frac{2 x}{\pi}} e^{-\frac{4 \pi^{2}}{k x}}
\end{aligned}
$$

which completes the proof of Theorem 2.2.

\section{Two asymptotic formulas found on page 45 of Ramanujan's lost notebook}

In this section, we use Theorem 2.2 to prove the two asymptotic formulas found on page 45 of Ramanujan's lost notebook [21]. First we prove a lemma which allows us to explicitly calculate $L(s, \chi)$, where $s=1,3,5,7$, and $\chi$ is odd.

Lemma 3.1. Let $\chi$ be a primitive real non-principal odd character mod $k$. Then

$$
\begin{aligned}
& L(1, \chi)=\frac{\pi i}{k^{2}} G(\chi) M_{1}(\chi), \\
& L(3, \chi)=\frac{2 \pi^{3} i}{3 k^{4}} G(\chi)\left(k^{2} M_{1}(\chi)-M_{3}(\chi)\right), \\
& L(5, \chi)=\frac{2 \pi^{5} i}{15 k^{6}} G(\chi)\left(\frac{7}{3} k^{4} M_{1}(\chi)-\frac{10}{3} k^{2} M_{3}(\chi)+M_{5}(\chi)\right), \\
& L(7, \chi)=\frac{4 \pi^{7} i}{315 k^{8}} G(\chi)\left(\frac{31}{3} k^{6} M_{1}(\chi)-\frac{49}{3} k^{4} M_{3}(\chi)+7 k^{2} M_{5}(\chi)-M_{7}(\chi)\right),
\end{aligned}
$$


where $G(\chi)$ is the Gaussian sum defined by

$$
G(\chi)=\sum_{n=1}^{k-1} \chi(n) e^{2 \pi i n / k}
$$

and

$$
M_{m}(\chi)=\sum_{n=1}^{k-1} \chi(n) n^{m}
$$

Proof. From [7, p. 33, Eq. (6.12)], for $\chi$ is odd,

$$
G(\chi) M_{m}(\chi)=-2 i k^{m+1} \sum_{j=0}^{m-1} \frac{m !}{(m-j) !}(2 \pi)^{-j-1} \cos \left(\frac{j \pi}{2}\right) L(j+1, \chi) .
$$

Using (3.3) we may calculate $L(2 n-1, \chi)$ for any positive integer $n$. Letting $m=1$ in $(3.3)$, we obtain

$$
L(1, \chi)=\frac{\pi i}{k^{2}} G(\chi) M_{1}(\chi)
$$

If $m=3$, we find from (3.3) and (3.4) that

$$
L(3, \chi)=\frac{2 \pi^{3} i}{3 k^{4}} G(\chi)\left(k^{2} M_{1}(\chi)-M_{3}(\chi)\right)
$$

If $m=5$ in $(3.3)$,

$$
G(\chi) M_{5}(\chi)=-2 i k^{6}\left\{\frac{1}{2 \pi} L(1, \chi)-\frac{5}{2 \pi^{3}} L(3, \chi)+\frac{15}{4 \pi^{5}} L(5, \chi)\right\}
$$

Thus, using (3.4) and (3.5) in (3.6), we deduce that

$$
L(5, \chi)=\frac{2 \pi^{5} i}{15 k^{6}} G(\chi)\left(\frac{7}{3} k^{4} M_{1}(\chi)-\frac{10}{3} k^{2} M_{3}(\chi)+M_{5}(\chi)\right)
$$

Similarly, the result

$$
L(7, \chi)=\frac{4 \pi^{7} i}{315 k^{8}} G(\chi)\left(\frac{31}{3} k^{6} M_{1}(\chi)-\frac{49}{3} k^{4} M_{3}(\chi)+7 k^{2} M_{5}(\chi)-M_{7}(\chi)\right)
$$

follows by taking $m=7$ in (3.3). This completes the proof of Lemma 3.1. 
Theorem 3.2. As $x \rightarrow 0+$,

$$
\frac{(3 x)^{1 / 3}}{1}-\frac{1}{1+e^{x}}-\frac{1}{1+e^{2 x}}-\frac{1}{1+e^{3 x}}-\cdots=\frac{\Gamma\left(\frac{1}{3}\right)}{\Gamma\left(\frac{2}{3}\right)} e^{G(x)},
$$

where

$$
G(x) \sim a_{2} x^{2}+a_{4} x^{4}+a_{6} x^{6}+\cdots,
$$

with

$$
a_{\nu}=\frac{4 \Gamma(\nu) \zeta(\nu) L(\nu+1, \chi)}{(2 \pi / \sqrt{3})^{2 \nu+1}}
$$

where $\chi(n)=\left(\frac{n}{3}\right)$. In particular,

$$
a_{2}=\frac{1}{108}, \quad a_{4}=\frac{1}{4320}, \quad \text { and } \quad a_{6}=\frac{1}{38880} .
$$

Furthermore, as $x \rightarrow 0+$,

$$
\text { the minimum value of } a_{\nu} x^{\nu} \sim \frac{3}{\pi} \sqrt{\frac{2 x}{\pi}} e^{-\frac{4 \pi^{2}}{3 x}} \text {. }
$$

Proof. The continued fraction on the left hand side of (3.7) is equivalent to

$$
\begin{aligned}
& (3 x)^{1 / 3}\left(\frac{1}{1}-\frac{1}{e^{x}\left(1+e^{-x}\right)}-\frac{1}{e^{2 x}\left(1+e^{-2 x}\right)}-\frac{1}{e^{3 x}\left(1+e^{-3 x}\right)}-\cdots\right) \\
= & (3 x)^{1 / 3}\left(\frac{1}{1}-\frac{e^{-x}}{1+e^{-x}}-\frac{e^{-3 x}}{1+e^{-2 x}}-\frac{e^{-5 x}}{1+e^{-3 x}}-\cdots\right) \\
= & (3 x)^{1 / 3} \frac{\left(e^{-2 x} ; e^{-3 x}\right)_{\infty}}{\left(e^{-x} ; e^{-3 x}\right)_{\infty}},
\end{aligned}
$$

by (1.2), which can be found in Ramanujan's second notebook [20] and which was first proved by Andrews, Berndt, Jacobsen, and Lamphere [5], [9, p. 46].

This expression is the case $k=3$ in Theorem 2.2 , since $M_{1}(\chi)=-1$. This completes the proof of (3.7) and (3.9).

To prove (3.8), we need the well-known values [18, pp. 776-777],

$$
\zeta(2)=\frac{\pi^{2}}{6}, \quad \zeta(4)=\frac{\pi^{4}}{90}, \quad \text { and } \quad \zeta(6)=\frac{\pi^{6}}{945},
$$

and the following values from Lemma 3.1 with $k=3$,

$$
L(3, \chi)=\frac{4 \pi^{3} \sqrt{3}}{243}, \quad L(5, \chi)=\frac{4 \pi^{5} \sqrt{3}}{3^{7}}, \quad \text { and } \quad L(7, \chi)=\frac{56 \pi^{7} \sqrt{3}}{3^{10} \cdot 5}
$$

since $G(\chi)=i \sqrt{3}, M_{1}(\chi)=-1, M_{3}(\chi)=-7, M_{5}(\chi)=-31$, and $M_{7}(\chi)=-127$. Therefore, the values in (3.8) now easily follow from (2.3). 
Theorem 3.3. As $x \rightarrow 0+$,

$$
\frac{2 \sqrt{x}}{1}-\frac{1}{e^{x}+e^{-x}}-\frac{1}{e^{2 x}+e^{-2 x}}-\frac{1}{e^{3 x}+e^{-3 x}}-\cdots=\frac{\Gamma\left(\frac{1}{4}\right)}{\Gamma\left(\frac{3}{4}\right)} e^{G(x)},
$$

where

$$
G(x) \sim a_{2} x^{2}+a_{4} x^{4}+a_{6} x^{6}+\cdots,
$$

with

$$
a_{\nu}=\frac{4 \Gamma(\nu) \zeta(\nu) L(\nu+1, \chi)}{\pi^{2 \nu+1}}
$$

where $\chi$ is the nonprincipal, primitive character modulo 4. Furthermore,

$$
a_{2}=\frac{1}{48}, \quad a_{4}=\frac{1}{1152}, \quad \text { and } \quad a_{6}=\frac{61}{362880},
$$

and, as $x \rightarrow 0+$,

$$
\text { the minimum value of } a_{\nu} x^{\nu} \sim \frac{4}{\pi} \sqrt{\frac{2 x}{\pi}} e^{-\frac{\pi^{2}}{x}} \text {. }
$$

Proof. By using equivalence relations and (1.3), we can write the continued fraction on the left hand side of (3.11) in the form

$$
\begin{aligned}
& 2 \sqrt{x}\left(\frac{1}{1}-\frac{1}{e^{x}\left(1+e^{-2 x}\right)}-\frac{1}{e^{2 x}\left(1+e^{-4 x}\right)}-\frac{1}{e^{3 x}\left(1+e^{-6 x}\right)}-\cdots\right) \\
= & 2 \sqrt{x}\left(\frac{1}{1}-\frac{e^{-x}}{1+e^{-2 x}}-\frac{e^{-3 x}}{1+e^{-4 x}}-\frac{e^{-5 x}}{1+e^{-6 x}}-\cdots\right) \\
= & 2 \sqrt{x} \frac{\left(e^{-3 x} ; e^{-4 x}\right)_{\infty}}{\left(e^{-x} ; e^{-4 x}\right)_{\infty}} .
\end{aligned}
$$

Equality (1.3) is in Ramanujan's second notebook [20], [9, p. 48]. It is also simply the case $a=1, b=0$ of Entry 12 in Chapter 16 of Ramanujan's second notebook [20], [8, p. 24]. Among others, K. G. Ramanathan [19] has given a proof of (1.3). Another continued fraction for the product on the left side of (1.3) is found in the "lost notebook" and has been proved by Andrews [3] as well as by Ramanathan $[19]$.

This expression (3.14) is the case $k=4$ in Theorem 2.2, since $M_{1}(\chi)=-2$. This completes the proof of (3.11) and (3.13).

¿From Lemma 3.1 with $k=4$, we find that

$$
L(3, \chi)=\frac{\pi^{3}}{32}, \quad L(5, \chi)=\frac{\pi^{5}}{4^{5} \cdot 15}, \quad \text { and } \quad L(7, \chi)=\frac{61 \pi^{7}}{3^{2} \cdot 5 \cdot 4^{6}},
$$

since $G(\chi)=2 i, M_{1}(\chi)=-2, M_{3}(\chi)=-26, M_{5}(\chi)=-242$, and $M_{7}(\chi)=-2186$. Hence, using (3.10) and the values above in (2.3), we readily compute the values in (3.12).

Ramanujan did not record the value of $a_{6}$.

We record two further corollaries, but we do not know any continued fractions represented by the products. 
Corollary 3.4. As $x \rightarrow 0+$,

$$
(6 x)^{\frac{2}{3}} \frac{\left(e^{-5 x} ; e^{-6 x}\right)_{\infty}}{\left(e^{-x} ; e^{-6 x}\right)_{\infty}}=\frac{\Gamma\left(\frac{1}{6}\right)}{\Gamma\left(\frac{5}{6}\right)} e^{G(x)},
$$

where

$$
G(x) \sim a_{2} x^{2}+a_{4} x^{4}+a_{6} x^{6}+\cdots,
$$

with

$$
a_{\nu}=\frac{4 \Gamma(\nu) \zeta(\nu) L(\nu+1, \chi)}{(2 \pi / \sqrt{6})^{2 \nu+1}},
$$

where $\chi$ is the nonprincipal, primitive character modulo 6. Furthermore, as $x \rightarrow$ $0+$,

$$
\text { the minimum value of } a_{\nu} x^{\nu} \sim \frac{6}{\pi} \sqrt{\frac{2 x}{\pi}} e^{-\frac{2 \pi^{2}}{3 x}} \text {. }
$$

Proof. This is the case $k=6$ in Theorem 2.2, since $M_{1}(\chi)=-4$.

Corollary 3.5. As $x \rightarrow 0+$,

$$
(7 x) \frac{\left(e^{-3 x}, e^{-5 x}, e^{-6 x} ; e^{-7 x}\right)_{\infty}}{\left(e^{-x}, e^{-2 x}, e^{-4 x} ; e^{-7 x}\right)_{\infty}}=\frac{\Gamma\left(\frac{1}{7}\right) \Gamma\left(\frac{2}{7}\right) \Gamma\left(\frac{4}{7}\right)}{\Gamma\left(\frac{3}{7}\right) \Gamma\left(\frac{5}{7}\right) \Gamma\left(\frac{6}{7}\right)} e^{G(x)},
$$

where

$$
G(x) \sim a_{2} x^{2}+a_{4} x^{4}+a_{6} x^{6}+\cdots,
$$

with

$$
a_{\nu}=\frac{4 \Gamma(\nu) \zeta(\nu) L(\nu+1, \chi)}{(2 \pi / \sqrt{7})^{2 \nu+1}},
$$

where $\chi$ is the nonprincipal, primitive, real character modulo \%. Furthermore, as $x \rightarrow 0+$,

$$
\text { the minimum value of } a_{\nu} x^{\nu} \sim \frac{7}{\pi} \sqrt{\frac{2 x}{\pi}} e^{-\frac{4 \pi^{2}}{7 x}} .
$$

Proof. This is the case $k=7$ in Theorem 2.2, since $M_{1}(\chi)=-7$.

\section{The case when $\chi$ is even}

In Theorem 2.2, we considered the case when $\chi$ is odd. In this section, we consider the case when $\chi$ is even. ¿From [12, p. 71] we know that

$$
L(s, \chi)=0,
$$

if $s=0,-2,-4,-6, \ldots$ Hence the integrand of (2.6) has simple poles only at $s=0$ and $s=-1$. Now if we follow the same steps as we did in the proof of Theorem 2.2 , we deduce that, for any $N>1$, as $x$ tends to $0+$,

$$
\log \prod_{n=1}^{k-1}\left(e^{-n x} ; e^{-k x}\right)_{\infty}^{-\chi(n)}=L^{\prime}(0, \chi)-\zeta(0) L(-1, \chi) x+O\left(x^{N}\right)
$$


¿From [13, p. 181, Eq. (3.2)], if $\chi$ is even,

$$
L^{\prime}(0, \chi)=\frac{k}{2 G(\chi)} L(1, \chi)
$$

where $G(\chi)$ is defined by (3.1). But from [13, p. 182, Eq. (3.5)],

$$
L(1, \chi)=-\frac{G(\chi)}{k} \sum_{n=1}^{k-1} \chi(n) \log \left|1-\zeta_{k}^{n}\right|
$$

where $\zeta_{k}=\exp (2 \pi i / k)$.

Hence, by (4.2) and (4.3),

$$
L^{\prime}(0, \chi)=-\frac{1}{2} \sum_{n=1}^{k-1} \chi(n) \log \left|1-\zeta_{k}^{n}\right|=\log \left(\prod_{n=1}^{k-1}\left|1-\zeta_{k}^{n}\right|^{-\frac{\chi(n)}{2}}\right)
$$

Since $\chi$ is an even character, the functional equation for $L(s, \chi)$ is given by $[12, \mathrm{p}$. $72]$

$$
L(s, \chi)=\left(\frac{\pi}{k}\right)^{s-\frac{1}{2}} \frac{\Gamma\left(\frac{1}{2}(1-s)\right)}{\Gamma\left(\frac{1}{2} s\right)} L(1-s, \chi) .
$$

By (4.5) and (2.16),

$$
L(-1, \chi)=\left(\frac{\pi}{k}\right)^{-\frac{3}{2}} \frac{\Gamma(1)}{\Gamma\left(-\frac{1}{2}\right)} L(2, \chi)=-\frac{k^{\frac{3}{2}}}{2 \pi^{2}} L(2, \chi) .
$$

¿From [7, p. 32, Eq. (6.10)],

$$
L(2, \chi)=\frac{\pi^{2}}{k^{3}} G(\chi) M_{2}(\chi)
$$

where $G(\chi)$ and $M_{2}(\chi)$ are defined by (3.1) and (3.2), respectively. Also $G(\chi)=\sqrt{k}$, since $\chi$ is even. Hence, from (4.6) and (4.7),

$$
L(-1, \chi)=-\frac{k^{\frac{3}{2}}}{2 \pi^{2}} \frac{\pi^{2}}{k^{3}} \sqrt{k} M_{2}(\chi)=-\frac{1}{2 k} M_{2}(\chi) .
$$

¿From [25, p. 19],

$$
\zeta(0)=-\frac{1}{2}
$$

Therefore, by (4.1), (4.4), (4.9), and (4.8), we obtain the following theorem. 
Theorem 4.1. Let $k$ be a positive integer greater than 3, and let $L(s, \chi)$ denote the Dirichlet L-function associated with $\chi(n)$, a primitive real non-principal even character mod $k$. Then as $x \rightarrow 0+$,

$$
\prod_{n=1}^{k-1}\left(e^{-n x} ; e^{-k x}\right)_{\infty}^{-\chi(n)} \sim\left(\prod_{n=1}^{k-1}\left|1-\zeta_{k}^{n}\right|^{-\frac{\chi(n)}{2}}\right) e^{-\frac{1}{4 k} M_{2}(\chi) x},
$$

where $\zeta_{k}=\exp (2 \pi i / k)$ and $M_{2}(\chi)$ is defined by (3.2).

By using Theorem 4.1, we may obtain asymptotic formulas for the RogersRamanujan and Göllnitz-Gordon continued fractions defined by (1.1) and (1.4), respectively.

The product representation for $R(q)$ given in (1.1) was originally discovered by L. J. Rogers [23] in 1894 and rediscovered by Ramanujan; see his notebooks [20, Chap. 16, Entry 38 (iii)], [8, p. 79].

On page 229 of his second notebook [20, p. 229], Ramanujan recorded a product representation for $H(q)$ given in (1.4). Without any knowledge of Ramanujan's work, H. Göllnitz [14] and B. Gordon [15] rediscovered and proved (1.4) independently. Shortly thereafter Andrews [2] proved (1.4) as a corollary of a more general result.

Corollary 4.2. As $x \rightarrow 0+$,

$$
R\left(e^{-x}\right) \sim \frac{\sqrt{5}-1}{2}
$$

Proof. Let $k=5$ in Theorem 4.1. Since $\cos (2 \pi / 5)=(\sqrt{5}-1) / 4$, we find by a straightforward calculation that

$$
\prod_{n=1}^{4}\left|1-\zeta_{5}^{n}\right|^{-\frac{\chi(n)}{2}}=\frac{\sqrt{5}+1}{2}
$$

where $\zeta_{5}=\exp (2 \pi i / 5)$.

Therefore,

$$
\frac{\left(e^{-2 x}, e^{-3 x} ; e^{-5 x}\right)_{\infty}}{\left(e^{-x}, e^{-4 x} ; e^{-5 x}\right)_{\infty}} \sim \frac{\sqrt{5}+1}{2} e^{-\frac{1}{5} x},
$$

since $M_{2}(\chi)=4$. This completes the proof by (1.1) and (4.10).

Corollary 4.2 was also proved by J. Lehner [17] by a different method.

Corollary 4.3. Recall that $H(q)$ is defined in (1.4). As $x \rightarrow 0+$,

$$
H\left(e^{-x}\right) \sim \sqrt{2}-1
$$

Proof. Let $k=8$ in Theorem 4.1. By a straightforward calculation,

$$
\prod_{n=1}^{7}\left|1-\zeta_{8}^{n}\right|^{-\frac{\chi(n)}{2}}=\sqrt{2}+1
$$


where $\zeta_{8}=\exp (\pi i / 4)$. Hence,

$$
\frac{\left(e^{-3 x}, e^{-5 x} ; e^{-8 x}\right)_{\infty}}{\left(e^{-x}, e^{-7 x} ; e^{-8 x}\right)_{\infty}} \sim(\sqrt{2}+1) e^{-\frac{1}{2} x},
$$

since $M_{2}(\chi)=16$. The result follows from (1.4) and (4.11).

\section{References}

1. M. ABRAMOWITZ and I. A. STEGUN, EDITORS, Handbook of Mathematical Functions, (Dover, New York, 1965).

2. G. E. ANDREWS, 'On $q$-difference equations for certain well-poised basic hypergeometric series', Quart. J. Math. (Oxford) 19 (1968), 433-447.

3. G. E. ANDREWS, 'Ramanujan's "lost" notebook III. The Rogers-Ramanujan continued fraction', Adv. in Math. 41 (1981), 186-208.

4. G. E. ANDREWS, R. ASKEY, and R. ROY, Special Functions, (University Press, Cambridge, 1999).

5. G. E. ANDREWS, B. C. BERNDT, L. JACOBSEN, and R. L. LAMPHERE, 'The continued fractions found in the unorganized portions of Ramanujan's notebooks', Memoir, American Mathematical Society No. 477, Providence, RI, 1992.

6. T. M. APOSTOL, Introduction to Analytic Number Theory, (Springer-Verlag, New York, 1976).

7. B. C. BERNDT, The Voronöi summation formula, (in The Theory of Arithmetic Functions, A. A. Gioia and D. L. Goldsmith, eds., Lecture Notes in Math. No. 251, Springer-Verlag, Berlin, 1972), pp. 21-36.

8. B. C. BERNDT, Ramanujan's Notebooks, Part III, (Springer-Verlag, New York, 1991).

9. B. C. BERNDT, Ramanujan's Notebooks, Part V, (Springer-Verlag, New York, 1998).

10. Z. I. BOREVICH and I. R. SHAFAREVICH, Number Theory, (Academic Press, New York, 1966).

11. E. T. COPSON, Theory of Functions of a Complex Variable, (Clarendon Press, Oxford, 1935).

12. H. DAVENPORT, Multiplicative Number Theory, 2nd ed., (Springer-Verlag, New York, 1980).

13. C. DENINGER, 'On the analogue of the formula of Chowla and Selberg for real quadratic fields', J. Reine Angew. Math. 351 (1984), 171-191.

14. H. GÖLLNITZ, 'Partitionen mit Differenzenbedingungen', J. Reine Angew. Math. 225 (1967), 154-190.

15. B. GORDON, 'Some continued fractions of the Rogers-Ramanujan type', Duke Math. J. 32 (1965), 741-748.

16. I. S. GRADSHTEYN and I. M. RYZHIK, eds., Table of Integrals, Series and Products, 5th ed., (Academic Press, San Diego, CA, 1994).

17. J. LEHNER, 'A partition function connected with the modulus 5', Duke Math. J. 8 (1941), 631-655.

18. A. P. PRUDNIKOV, YU. A. BRYCHKOV, and O. I. MARICHEV, Integrals and Series, Vol. 1: Elementary Functions, (Gordon and Breach, Amsterdam, 1986).

19. K. G. RAMANATHAN, 'Ramanujan's continued fraction', Indian J. Pure Appl. Math. 16 (1985), 695-724.

20. S. RAMANUJAN, Notebooks (2 volumes), (Tata Institute of Fundamental Research, Bombay, 1957).

21. S. RAMANUJAN, The Lost Notebook and Other Unpublished Papers, (Narosa, New Delhi, 1988).

22. L. RICHMOND and G. SZEKERES, 'The Taylor coefficients of certain infinite products', Acta Sci. Math. 40 (1978), 347-369. 
23. L. J. ROGERS, 'Second memoir on the expansion of certain infinite products', Proc. London Math. Soc. 25 (1894), 318-343.

24. E. C. TITCHMARSH, Theory of Fourier Integrals, 3rd ed., (Chelsea Publishing Company, New York, 1986).

25. E. C. TITCHMARSH, The Theory of the Riemann Zeta-Function, Clarendon Press, (Oxford, 1951).

Department of Mathematics, 1409 West Green Street, University of Illinois, URBANA, IL 61801, USA

E-mail address: berndt@math.uiuc.edu

Department of Mathematics, 1409 West Green Street, University of Illinois, URBANA, IL 61801, USA

Current address: Department of Mathematics, 231 W. 18th Avenue, The Ohio State University, Columbus, OH 43210, USA

E-mail address: sohn@math.ohio-state.edu 